\title{
A New Application of the Fixed-Points Theory for the Control of Kinetic Energy of a Continuous Structure
}

\author{
Jedol Dayou ${ }^{\dagger}$ \\ Vibration and Sound Research Group (VIBS), School of Science and Technology, \\ University of Malaysia Sabah, Locked Bag 2073, 88999 Kota Kinabalu, Sabab, Malaysia
}

(Received 30 August 2004; accepted 11 November 2005)

\begin{abstract}
The fixed-points theory was originally used to determine the optimum tuning and damping ratios of a vibration neutraliser that may flatten the frequency response function (FRF) of a simple structure. Although the theory has also been used for the control of vibration of a continuous structure, applications have been limited only to point control. In this paper, a new application of the theory is discussed, which is for the global control of vibration of a continuous structure. The theory is used to determine the optimum tuning and damping ratios of the vibration neutraliser that flatten the global response of the structure. Kinetic energy is used as a measure of the structure's global response, and the application is demonstrated on undamped and damped simply supported beams. It is found that by using these optimum values, it is possible to remove the effects of the dominant mode leaving only the effect of the residual modes in the global behaviour. This shows that the theory can also be used to reduce the vibration of a continuous structure at all points instead of at a particular point only, such as in the conventional application.
\end{abstract}

${ }^{\dagger}$ Member of the International Institute of Acoustics and Vibration (IIAV)

\section{INTRODUCTION}

A new application of the fixed-points theory is discussed. The theory is used to determine the optimum tuning and damping ratios of the vibration neutraliser that can be used to flatten the global behaviour of a continuous structure for both undamped and damped cases.

The vibration neutraliser has been used in many applications since it was invented in 1909., ${ }^{1,2}$ Applications range from small handheld tools, such as electrical clippers, ${ }^{3}$ to giant structures such as high-rise buildings ${ }^{4,5}$ and bridges, ${ }^{6}$ to large moving structures, such as aircraft. ${ }^{7,89}$ The vibration neutraliser was designed in such a way that its natural frequency coincides with either 1) the problematic resonance frequency of the host structure or 2) the frequency of disturbance away from the resonance region. ${ }^{10}$ In either case, the vibration neutraliser becomes less effective and may even increase the vibration amplitude of the host structure when there is a change in the frequency of the excitation force.

Previously, the fixed-points theory was used to determine the optimum tuning and damping ratios of a vibration neutraliser that flatten the frequency response function (FRF) of a simple structure. The theory has its roots dating back to 1932 when Hahnkamm ${ }^{11,2}$ suggested that the desired natural frequency of the vibration neutraliser should be given by

$$
f_{n_{\text {opt }}}=\frac{f_{h}}{1+\mu}
$$

where $f_{n_{\text {opt }}}$ is the neutraliser's optimal natural frequency, $f_{h}$ is the host's resonance frequency, and $\mu$ is the ratio between the neutraliser's and the host's mass. Later, Brock ${ }^{12}$ derived the expression for the neutraliser's optimal damping ratio, which can be written as

$$
\zeta_{n_{\text {opt }}}=\sqrt{\frac{3 \mu}{8(1+\mu)}},
$$

where $\zeta_{n}$ is the neutraliser's damping ratio defined as $\zeta_{n}=c_{n} /\left(2 M_{n} f_{n}\right) ; c_{n}, M_{n}$, and $f_{n}$ are the neutraliser's damping, mass, and natural frequency, respectively. Using these optimum values, the FRF curve of the composite system can be made relatively flat with no new resonance occurring.

The fixed-points theory was found to be very useful and has been used as one of the design laws when fabricating a vibration neutraliser. ${ }^{13}$ However, the applications of the theory have been limited only to relatively simple structures. The theory has also been used for continuous structures with well-separated natural frequencies, such as beams, but only in regards to point control problems. ${ }^{14}$ These are the conventional applications of the theory.

In this paper, the application of the theory is extended to a new area, which is for the control of global responses of a continuous structure. Kinetic energy is used as a measure of the global responses, and the theory is applied to determine the optimum tuning and damping ratios of the control device. It is found that the theory can be used to derive the optimum value of these parameters that flatten the FRF of the structure in terms of its kinetic energy. Therefore, it is shown that the theory can also be used to reduce the vibration of a continuous structure at all points instead of only at a particular point as in conventional applications. The new application is demonstrated on a simply supported beam as a host structure, for both undamped and damped cases, through some computer simulations. 